\title{
Comparación de sutura mecánica con sutura manual de la faringe en laringectomía total
}

\section{Comparison of mechanical suture with manual suture of the pharynx in total laryngectomy}

\author{
Jaime Osorio M. ${ }^{1,2}$, Carlos Morales A. ${ }^{1}$, Pilar Contreras R. ${ }^{1}$, Rodrigo Pineda D. ${ }^{1}$, Fernanda Silva R. ${ }^{1}$, \\ Daniel Muñoz S. ${ }^{3}$, Angélica Suazo D. ${ }^{4}$, Miguel Cancino C. ${ }^{1,2}$, Rodrigo Arregui $V^{1,2}$
}

\section{Resumen}

Introducción: La sutura mecánica es una opción para el cierre de la faringorrafia en laringectomía total por cáncer de laringe. Objetivo: Comparar el uso de sutura mecánica lineal con sutura manual de la faringe durante la laringectomía total por cáncer de laringe. Material y Método: Se realizó un estudio de tipo experimental prospectivo. Se compararon dos grupos pacientes: Los pacientes con sutura mecánica desde 2018 a marzo de 2020 y los pacientes con sutura manual previa a enero de 2018 en el Servicio de Otorrinolaringología del Hospital Barros Luco. Se analizaron fístula faringo-cutánea posoperatoria (FFC), tiempo operatorio, estadía hospitalaria y costo. Resultados: El estudio incluyó a 14 pacientes, cada grupo con $n=7$. El grupo con sutura mecánica presento $0 \%$ de FFC y el grupo sutura manual $28 \%$. El grupo con sutura mecánica reinicio alimentación a los 7 días y el grupo con sutura manual en promedio a los 11,5 (7-23) días. $(\mathrm{p}=0,0023)$. El tiempo promedio de cirugía para el grupo experimental es de $288 \pm 37,4$ minutos y con sutura manual $311 \pm 32,4$ minutos. $(p=0,0176)$. El promedio de hospitalización para el grupo experimental fue de $11 \pm 2,6$ días ( 9 a 16), y para el grupo control fue de $21 \pm 14$ días (10 a 49) ( $p<0,0001)$. Conclusión: La sutura mecánica es un procedimiento fácil de usar y seguro. Existiría un beneficio en el uso de sutura mecánica para el cierre faríngeo al compararlo con la sutura manual al disminuir el número de FFC, el tiempo operatorio y los días de hospitalización.

Palabras clave: Sutura mecánica, fístula faringo-cutánea, laringectomía total, faringorrafia, cáncer de laringe.

\begin{abstract}
Introduction: Mechanical suture is an option for the closure of the pharyngorrhaphy in total laryngectomy due to laryngeal cancer. Aim: To compare the use of linear mechanical suture with manual pharyngeal suture during total laryngectomy for laryngeal cancer. Material and Method: A prospective experimental study was carried out. Two patient groups were compared: patients with mechanical suture from 2018 to March 2020 and patients with manual suture prior to January 2018 at the otorhinolaryngology service of the Barros Luco Hospital. Postoperative pharyngocutaneous fistula (FFC), operative time, hospital stay and cost were analyzed. Results: The study included 14 patients, each group with $n=7$. The group with mechanical suture presented $0 \%$ of FFC and the group with manual suture $28 \%$. The group with mechanical suture restarted feeding at 7 days and the group with manual suture on average at 11.5 (7-23) days ( $p=0.0023)$. The average surgery time for the experimental group was 288 \pm 37.4 minutes, while for the group with manual suture was $311 \pm 32.4$ minutes $(p=0.0176)$. The mean hospitalization for the experimental group was $11 \pm 2.6$ days ( 9 to 16), and for the control group it was $21 \pm 14$ days (10 to 49) ( $p<0.0001)$. Conclusion: The mechanical suture is an easy to use and safe procedure. There would be a benefit in the use of mechanical suture for pharyngeal closure when buying it with manual suture by reducing the number of FFCs, operative time and days of hospitalization.
\end{abstract}

Keywords: Mechanical suture, pharyngocutaneous fistula, total laryngectomy, pharyngorraphy, laryngeal cancer.

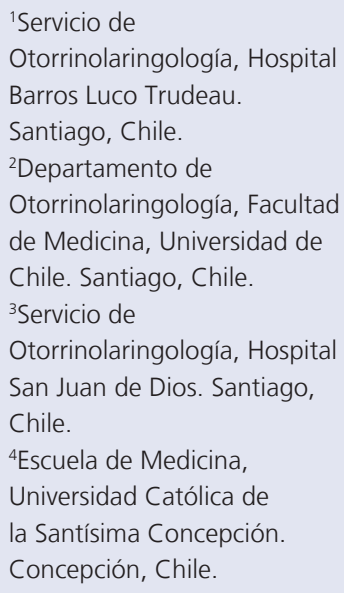

Los autores declaran no tener conflictos de interés. Este trabajo recibió financiamiento de fondos del concurso anual de investigación de la Sociedad Chilena de Otorrinolaringología y Cirugía de cabeza y Cuello.

Recibido el 1 de noviembre de 2020. Aceptado el 14 de marzo de 2021.

\section{Correspondencia:}

Dr. Jaime Osorio M. Gran Avenida José Miguel Carrera 3204, San Miguel. Santiago, Chile. Email: osorio.orl@gmail.com 


\section{Introducción}

En Chile, el cáncer de laringe es la primera causa de muerte en tumores malignos de cabeza y cuello, representando a un $25 \%$ de estos ${ }^{1}$. La incidencia reportada a nivel mundial es 0,5/100.000 habitantes en mujeres, 3,9/100.000 en hombres, mientras que en Chile es de 0,2/100.000 en mujeres, y 1,8/100.000 en hombres $^{1}$. La tasa de mortalidad bruta en nuestro país reportada en el 2012, fue de 0,9/100.000 habitantes, con una mortalidad específica que va de 0,04/100.000 en menores de 50 años, a $10,97 / 100.000$ en mayores de 80 años; con una razón hombre:mujer de 6:1,2.

El hábito tabáquico y el alcohol son los factores de riesgo más frecuentemente asociados con el desarrollo de cáncer laríngeo. El tabaquismo se asocia en un $97,2 \%$ con esta patología. El riesgo relativo de desarrollar cáncer de laringe en fumadores de 10 cigarrillos/día durante 10 años es 30 veces mayor que en no fumadores ${ }^{3}$. El número de cigarrillos y el fumar cigarrillos sin filtro son factores que aumentan la incidencia, lo que, unido a una consulta tardía, provoca que más de la mitad de los pacientes sea diagnosticado en una etapa avanzada, lo cual concuerda con la realidad observada en el Hospital Barros Luco y en el Hospital San Juan de Dios de Santiago en Chile ${ }^{3,4}$.

El objetivo del tratamiento es la erradicación de la enfermedad con la conservación de órganos, cuando es posible. En tumores glóti$\cos \mathrm{T} 1$ y T2 la cirugía y la radioterapia tienen excelentes resultados, con una sobrevida global a cinco años de $85 \%$ a $95 \%$ a 5 años, mientras que para las lesiones T3 independiente del tipo tratamiento (con o sin conservación de órgano) la sobrevida es cercano al $64 \%$ a 5 años y lesiones T4a la sobrevida es de un $40 \%$ a 5 años ${ }^{2}$.

La laringectomía total es actualmente el gold standard en el tratamiento para cáncer de laringe en estados avanzados, sin embargo, es un procedimiento no exento de complicaciones ${ }^{4}$. Las complicaciones mayores médicas son la embolia pulmonar, infarto de miocardio, accidentes cerebrovasculares y necesidad de ventilación mecánica. Mientras que, las quirúrgicas son la fístula faringocutánea, hemorragia posoperatoria, erosión carotídea y fístula linfática.
Dentro de estas complicaciones, una de las más frecuentes es la aparición de fístulas faringocutáneas (FFC), cuya incidencia es aproximadamente de un $12,5 \%$ según lo descrito en la literatura nacional, sin embargo, se describen resultados dispares como aquellas que cifran esta misma incidencia entre un 7,6\% y un $50 \%$. Otras series reportan cuando la laringectomía total fue el único tratamiento, la incidencia de fístulas alcanzó el 20\%, mientras que cuando se asociaron otros procedimientos, la incidencia fue del 34,5\%. El tiempo de hospitalización promedio de los pacientes fistulizados es de 23 días $^{5,6}$.

Las FFC posoperatoria pueden estar vinculada a diferentes factores generales, locales, radioterapia previa, estado nutricional del paciente y tipo de sutura ${ }^{6}$. La sutura mecánica es el método de cierre o anastomosis de los bordes de una herida mediante instrumentos semiautomáticos de diferentes diseños, características y longitudes, que utiliza la grapa con aparatos activados por la mano del cirujano ${ }^{7}$. La aparición de sutura mecánica semiautomática, que utilizan la grapa de titanio, puede constituir un motivo de progreso en la prevención de las complicaciones ya que las grapas de titanio tienen una excelente tolerancia por parte de los tejidos con mínima reacción inflamatoria, cierre seguro y hemostático, con pequeño daño tisular ${ }^{8}$.

La historia de la sutura mecánica comienza a principio de siglo XX, y no es hasta 1971 en que Lukyanchenko informó en la literatura rusa la primera experiencia clínica en laringectomía utilizando un dispositivo de grapado. Este sistema es frecuentemente utilizado en Europa del este, no así en occidente, donde sólo se reportan series de casos a partir de la década del $90^{9}$.

Actualmente existen los siguientes tipos de instrumentos de sutura mecánica: 1) sutura lineal tóraco-abdominal; 2) sutura circular; 3) división y ligadura; 4) cierre de fascias y piel'. La ventaja que tendría el uso de sutura mecánica lineal en la faringorrafia sería el de reducir los tiempos quirúrgicos y con ello la morbilidad del paciente, además de la disminución de complicaciones posoperatorias, como ya ha sido reportado en la literatura internacional. Si esto fuese así, podríamos reducir los días de estadía hospitalaria de nuestros pacientes, 
y con esto también, los costos y utilización de recursos que conlleva un paciente con una fístula faringocutánea ${ }^{7,10-12}$.

\section{Objetivo}

Comparar el uso de sutura mecánica lineal con sutura manual de la faringe durante la laringotomía total por cáncer de laringe. Específicamente, se pretende determinar y comparar la tasa de fístulas faringocutáneas, el reinicio de alimentación oral, el tiempo operatorio, el periodo de hospitalización y el costo de sutura mecánica de la faringe en la laringectomía total, con respecto a la técnica manual de sutura.

\section{Material y Método}

Se realizó un estudio de tipo experimental prospectivo, en donde el grupo experimental fue constituido por los pacientes sometidos a laringectomía total por cáncer de laringe que usaron sutura mecánica para la faringorrafia entre el periodo de enero de 2018 a marzo de 2020 y el grupo control a los paciente sometidos a laringectomía total por cáncer de laringe con sutura manual de la faringe previa a enero de 2018 en el Servicio de Otorrinolaringología del Hospital Barros Luco con la mismas características clínicas y epidemiológicas que el grupo experimental.

Los criterios inclusión fueron pacientes con indicación quirúrgica de laringectomía total por cáncer laríngeo y que haya firmado consentimiento informado para ingresar al estudio. Los criterios de exclusión fueron pacientes con cáncer laríngeo con indicación de uso de colgajos para corregir defecto dado la extensión tumoral, pacientes con cáncer de laringe con compromiso de uno de los senos piriformes o vallécula, paciente que hayan recibido radioterapia previa, pacientes que rechacen ser parte del estudio.

Se excluyeron los pacientes con compromiso de los senos piriformes o vallécula por ser una contraindicación para el uso de sutura mecánica con técnica cerrado o semicerrada. Se utilizo la técnica quirúrgica semicerrada para la faringorrafia con sutura mecánica lineal $80 \mathrm{~mm}(3,8 \mathrm{~mm} \times 1,5 \mathrm{~mm})$ de Purple Surgical, la cual es una grapadora que coloca dos hileras dobles de grapas de titanio alternas y paralelas y corta en el medio dividiendo las dos hileras (Figura 1). Una vez esqueletizado el marco laríngeo y separado de la tráquea y de la pared anterior del esófago, la laringe queda conectada a la hipofaringe por la mucosa y submucosa (Figura 2). Luego se presta especial atención en ubicar la epiglotis, se realiza una incisión sobre epiglotis se toma con un gancho para levantarla y no quede en medio de la sutura (Figura 3).

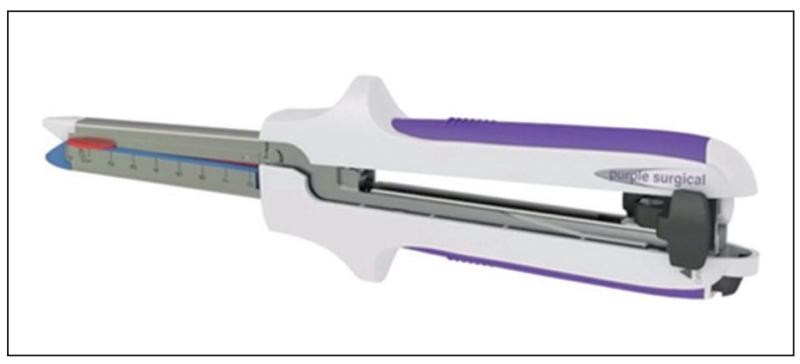

Figura 1. Sutura mecánica línea $80 \mathrm{~mm}$.

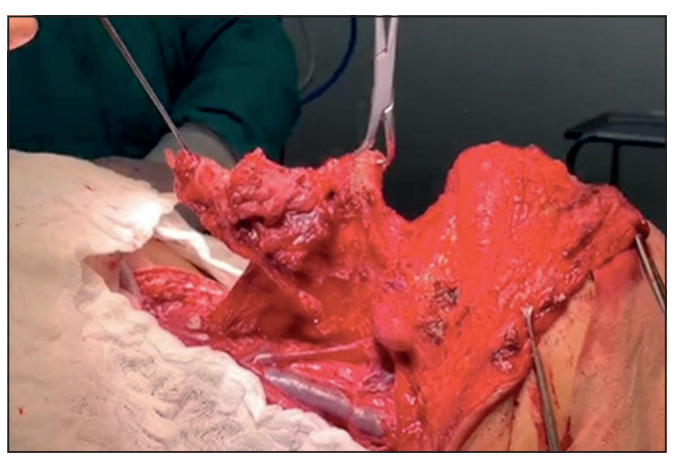

Figura 2. Esqueletización de la laringe.

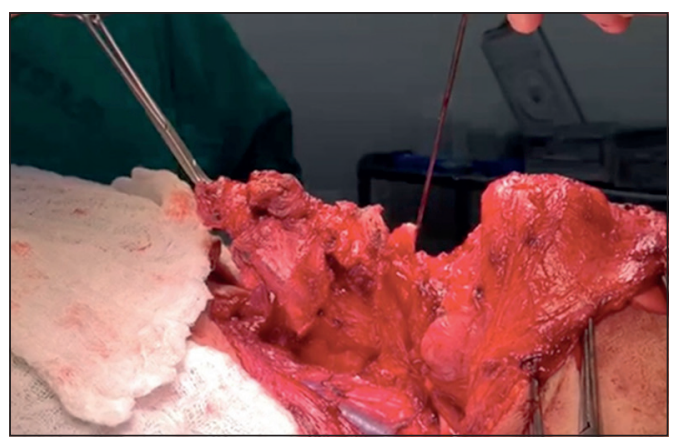

Figura 3. Técnica semicerrada. Se toma epiglotis a través de un ojal con un gancho. 
Se coloca el dispositivo de sutura mecánica entre la laringe y la hipofaringe. Se coloca el dispositivo de forma horizontal lo más cerca posible de la laringe para evitar la disección innecesaria de la mucosa faríngea. Luego el dispositivo se activa generando el grapado y luego el corte (Figura 4). Finalmente se revisa la faringorrafia y pieza quirúrgica para evaluar el margen quirúrgico macroscópico (Figuras 5 y 6$)$.

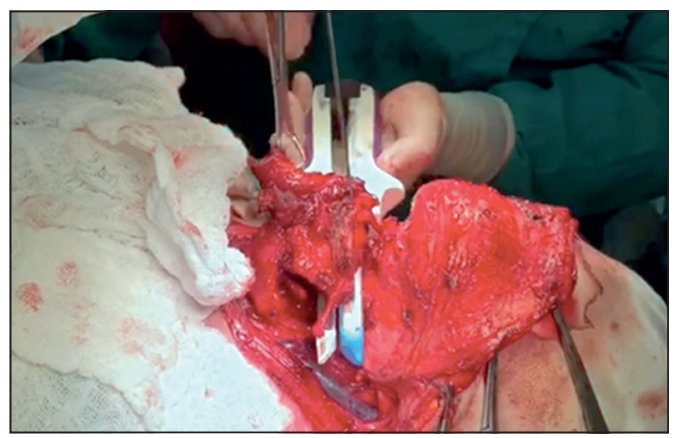

Figura 4. Se coloca la sutura mecánica en forma horizontal.

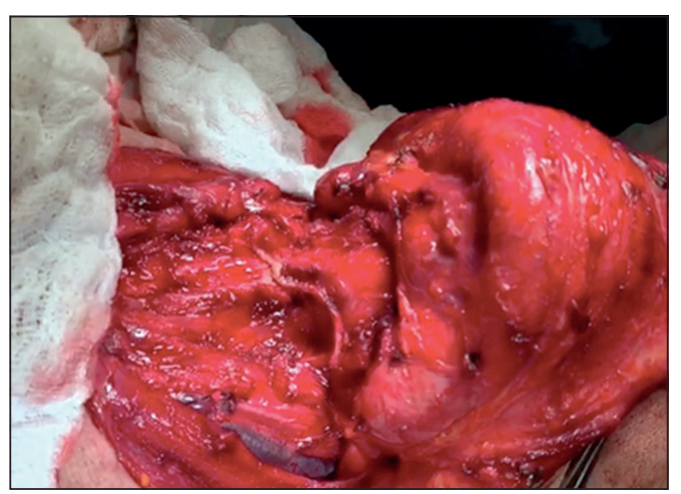

Figura 5. Línea de sutura horizontal.

Figura 6. Laringe, pieza quirúrgica.
La diferencia con la técnica con sutura manual es que se utiliza Sutura Vicryl 3/0 (poliglactina 910) con punto separado submucoso. En todos los pacientes se realizó vaciamiento ganglionar de cuello. Disección selectiva en $\mathrm{N} 0$ y disección radical modificada a $\mathrm{N}+$ para el manejo del cuello. A todos los pacientes en la cirugía se les coloca sonda nasogástrica para su alimentación posoperatoria y luego a los 7 días se realiza prueba de deglución con azul de metileno para descartar fístula faringocutánea si esta es negativa se retira sonda y se inicia alimentación oral.

Se realizó el análisis de subgrupo según edad, sexo, tamaño tumoral, comorbilidades, tabaquismo, índice paquete año (IPA), hemoglobina posoperatoria y albúmina preoperatoria, para determinar si son comparables, los sujetos fueron pareados por puntaje de propensión.

Los resultados analizados fueron la aparición de fístula faringocutánea, tiempo operatorio, estadía hospitalaria y costos asociados al procedimiento. Las variables cuantitativas se describen mediante promedio, desviación estándar (DE) y rango según corresponda. Se compararon mediante prueba de Wilcoxon. Se considerará como significativo un valor de $\mathrm{p}<0.05$ y todas las dócimas de hipótesis serán de dos colas estadísticas. Se utilizó el software estadístico Stata 16.

Se consideró la detención del estudio y análisis interno en caso de que en el grupo experimental aparezcan complicaciones superiores a las reportadas en la literatura para la técnica de sutura clásica. Este trabajo cuenta con la aprobación de Comité de Ética del Hospital Barros Luco, Número 557/2016 y todos los procedimientos satisfacen las normas éticas establecidas.

\section{Resultados}

El estudio incluyo 14 pacientes, cuya edad promedio fue de $70 \pm 6,8$ años, $100 \%$ hombres. Cada grupo de estudio tuvo siete pacientes $(\mathrm{n}=7)$. En la Tabla 1 se observan las características de cada grupo, mostrando que los grupos son comparables acorde puntaje de propensión. La Tabla 2 muestra un resumen de los resultados, según variable. 


\section{Fístula faringocutánea}

En el grupo con sutura mecánica se encontró un $0 \%$ de fístula a los 7 días. Mientras que, el grupo con sutura manual se encontró $28 \%(\mathrm{n}=2)$ de fístula a los 7 días. No se realiza dócima de hipótesis por frecuencia nula en grupo con sutura mecánica.

\section{Reinicio de alimentación}

En el grupo con sutura mecánica se inició su alimentación por boca en el $100 \%$ a los 7 días. En el grupo con sutura manual el inicio de su alimentación en promedio a los 11,5 (723). Se encontró una diferencia significativa con $\mathrm{p}=0,0023$.

\section{Tiempo promedio de cirugía}

El tiempo promedio de cirugía de laringectomía total más vaciamiento de cuello bilateral para el grupo experimental es de $288 \pm 37,4$ minutos y con sutura manual $311 \pm 32,4$ minutos. Se encontró una diferencia significativa $\operatorname{con} \mathrm{p}=0,0176$.

\section{Tiempo promedio de hospitalización}

El promedio de hospitalización para el grupo experimental fue de $18 \pm 17$ días con un rango de 9 a 56. El promedio de hospitalización para el grupo control fue de $21 \pm 14$ días con un rango de 10 a 49 . No siendo significativa esta diferencia. Al retirar al paciente de 56 días de hospitalización de grupo experimental que correspondió a una complicación por hematoma cervical el promedio baja a $11 \pm 2,6$ días rango de 9 a 16. Obteniendo una diferencia significativa con una $\mathrm{p}<0,0001$.
Tabla 1. Comparación de grupos estudiados con sutura manual y sutura mecánica. Según el método de pareamiento por puntaje de propensión, los grupos son comparables

$\begin{array}{cc}\begin{array}{c}\text { Sutura } \\ \text { manual } \\ (n=7)\end{array} & \begin{array}{c}\text { Sutura } \\ \text { mecánica } \\ (n=7)\end{array}\end{array}$

Edad

Promedio (DE) $69(7) \quad 71(7)$

Sexo

Masculino $\quad 100 \%(7) \quad 100 \%(7)$

Diabetes $\quad 28 \%(2) \quad 28 \%(2)$

Hipertensión $\quad 57 \%(4) \quad 71 \%(5)$

$\begin{array}{lll}\text { Asma } & 14 \%(1) & 0 \%\end{array}$

Tabaquismo $\quad 100 \%(7) \quad 100 \%(7)$

IPA (DE) $\quad 53(20) \quad 39(20)$

T3 $3 \quad 3$

$\begin{array}{lll}\mathrm{T} 4 & 4 & 4\end{array}$

$\mathrm{N}+\quad 4 \quad 3$

Sitio primario

Supraglótico $\quad 2$

Glótico 11

Transglóticos $\quad 4 \quad 5$

Hemoglobina posoperatoria (DE) $\quad 12,8(2,01) \quad 12,2(4,14)$

Albúmina preoperatoria (DE) $3,65(0,68) \quad 5,5(3,02)$

Márgenes quirúrgicos positivos $\quad 1$

Tabla 2. Resumen de resultados encontrados

\begin{tabular}{lccc}
\hline & Sutura mecánica & Sutura manual & P* \\
\hline Fístula faringocutánea & 0 & 2 & $p=0,0176$ \\
Tiempo promedio de cirugía & $288 \pm 37,4$ & $311 \pm 32,4$ & $p=0,0023$ \\
\hline Inicio de alimentación por boca & 7 & $11,5(7-23)$ & $p<0,0001$ \\
Días de hospitalización & $11 \pm 2,6$ & $21 \pm 14$ & - \\
\hline Costo & $\$ 95.000$ & $\$ 9.289$ & \\
\hline${ }^{*}$ prueba de Wilcoxon. & & & \\
\hline
\end{tabular}




\section{Costo}

A la fecha del estudio, el valor es la sutura mecánica tipo stapler lineal T.A. $80 \mathrm{~mm}$ Purple Surgical es de $\$ 95.000$ CLP y la sutura Vicryl $3 / 0$ SH considerando tres unidades es de $\$ 9.289$ CLP, por cirugía. Día cama 39.430 CLP en el Hospital Barros Luco.

\section{Discusión}

En la literatura se describen varias técnicas quirúrgicas (cerrada ${ }^{7}$, semicerrada ${ }^{10} y$ abierta $^{12}$ ) para realizar la faringorrafia con sutura mecánica posterior a laringectomía total. Se prefirió en este trabajo realizar la técnica semicerrada para tener control de la epiglotis y de esta manera, evitar el cruce con la línea de sutura. Otros centros con mayor experiencia prefieren realizar la técnica abierta con mayor control de los bordes quirúrgicos, pero se pierde el sello con salida de saliva al campo operatorio. La evaluación preoperatoria de la extensión $\mathrm{y}$ áreas comprometidas es fundamental para elegir la técnica con sutura mecánica, ya que al haber compromiso de vallécula o senos piriformes se debe elegir sutura mecánica con técnica abierta para evitar márgenes positivos. En nuestro estudio descartamos los pacientes con compromiso de los senos piriformes y vallécula para poder comparar mejor los dos grupos $^{13}$.

En una revisión sistemática se reportó un porcentaje de FFC menos con sutura mecánica que con sutura manual $(8,7 \% \text { vs. } 22,9 \%)^{5}$. En nuestro centro el porcentaje de fístulas faringocutáneas entre el años 2008 al 2018 fue de un $21,7 \%$ ( 15 de 70 pacientes) y al analizar los paciente que no recibieron radioterapia previa a la cirugía el porcentaje de fístula baja a 15,9\% de 64 pacientes ${ }^{14}$. En nuestro estudio identificamos una diferencia de FFC entre los grupos estudiados a favor de la sutura mecánica. El hecho de no tener FFC con el uso de sutura mecánica se puede explicar por el tamaño de la muestra del grupo de pacientes reclutados ${ }^{15}$.

El tiempo de reinicio de alimentación oral fue de 4 días menos en promedio para los pacientes con sutura mecánica debida ausencia de FFC, lo que coincide con la tendencia encontrada en la literatura en donde en promedio disminuyó 8 días 5 . El promedio de tiempo de cirugía fue 22 minutos inferior para los pacientes operados con sutura mecánica. En la literatura, se puede observar una reducción entre 45 a 80 minutos de cirugía ${ }^{5,15}$, lo que incide directamente en la morbilidad y mortalidad perioperatoria.

El tiempo de hospitalización es menor en 3 días en promedio para la sutura mecánica porque las FFC extiende el tiempo de hospitalización, pero la diferencia se amplía a 11 días, al sacar al paciente del grupo de sutura mecánica con un hematoma cervical que extendió 56 días su estadía hospitalaria por otros motivos ${ }^{5}$. $\mathrm{Al}$ analizar los costos, la sutura mecánica es 10 veces más cara que la sutura manual, pero la sutura manual presentaría más FFC que la sutura mecánica y por cada paciente con FFC solo por concepto de hospitalización se gasta $\$ 591.450$ CLP sin considerar medicamentos, alimentación enteral ni insumos. De la misma forma, menos tiempo operatorio y menos complicaciones con la sutura mecánica disminuye los costos totales. Está fuera del objetivo de nuestro trabajo, pero sería interesante conocer el costo beneficio del uso de sutura mecánica en laringectomía. Las limitaciones de nuestro estudio son el bajo número de pacientes, la comparación con una cohorte retrospectiva, y la falta de aleatorización.

\section{Conclusión}

Existiría un beneficio en el uso de sutura mecánica para el cierre faríngeo al comprarlo con la sutura manual al disminuir el número de FFC, el tiempo operatorio y los días de hospitalización. La técnica con sutura mecánica en laringectomía total por cáncer de laringe es un procedimiento fácil de aplicar y seguro, por lo que es una herramienta que debe ser considerada por el cirujano al momento de la faringorrafia.

\section{Bibliografía}

1. Cardemil F, Ortega G, Cabezas L. Importancia de la epidemiología en cáncer laríngeo: Incidencia y mortalidad por carcinoma escamoso de laringe. Rev Otorrinolaringol Cir Cabeza Cuello. 2017;77: 107-112. 
2. Yañez L, Loyola F, Cornejo J. Laringectomía total ampliada en carcinoma laríngeo avanzado T4a. Rev Chilena de Cirugía. 2012;64(5):442-446.

3. Pradenas M, Vallejos M, Rahal M, Cisternas A. Cáncer de laringe e hipofaringe experiencia HBLT 1990-2000. Rev Otorrinolaringol Cir Cabeza Cuello. 2004;64:13-2.

4. Contreras JM, Niklitschek B, Paredes A, Maass JC. Cáncer laríngeo-hipofaríngeo. Evaluación en el Hospital San Juan de Dios. Rev Otorrinolaringol Cir Cabeza Cuello. 2001; 61:19-30.

5. Aires FT, Dedivitis RA, Castro MA, Bernardo WM, Cernea CR, Brandão LG. Efficacy of stapler pharyngeal closure after total laryngectomy: A systematic review. Head Neck. 2014;36(5):739-742. doi: 10.1002/hed.23326.

6. Santaolalla Montoya F, Ruiz de Galarreta JC, Sánchez del Rey A, Martínez Ibargüen A, Zabala López de Maturana A. Estudio comparativo entre el empleo de la sutura manual y la sutura mecánica en el cierre del defecto mucoso en la laringectomía total [Comparative study of the use of manual and mechanical suturing in the closure of the mucosal defect in total laryngectomy]. Acta Otorrinolaringol Esp. 2002;53(5):343-350. doi: 10.1016/s00016519(02)78319-2.

7. Bedrin L, Ginsburg G, Horowitz Z, Talmi YP. 25-year experience of using a linear stapler in laryngectomy. Head Neck. 2005;27(12):1073-1079. doi: 10.1002/hed.20280.

8. Sarra L, Rodríguez J, García Valea M, Bitar J, Da Silva A. Fístula tras laringectomía total. Estudio retrospectivo y revisión bibliográfica. Acta Otorrinolaringol Esp. 2009;60(3):186-9.
9. Barrera A, Bannura G, Illanes F, Melo F. Anastomosis terminal funcional con sutura mecánica en cirugía intestinal. Resultados precoces de una serie prospectiva. Rev Chilena de Cirugía. 2012;64(3):274277.

10. Altissimi G, Frenguelli A. Linear stapler closure of the pharynx during total laryngectomy: a 15year experience (from closed technique to semiclosed technique). Acta Otorhinolaryngol Ital. 2007;27(3):118-122.

11. Sofferman RA, Voronetsky I. Use of the linear stapler for pharyngoesophageal closure after total laryngectomy. Laryngoscope. 2000;110(8):1406-1409. doi: 10.1097/00005537-200008000-00035.

12. Talmi YP, Finkelstein Y, Gal R, Shvilli Y, Sadov R, Zohar Y. Use of a linear stapler for postlaryngectomy pharyngeal repair: a preliminary report. Laryngoscope. 1990;100(5):552-555. doi: 10.1288/00005537199005000-00021.

13. Miles BA, Larrison D, Myers LL. Comparison of complication rates associated with stapling and traditional suture closure after total laryngectomy for advanced cancer. Ear Nose Throat J. 2013;92(8):392399. doi: 10.1177/014556131309200815.

14. Nicolas A, Abarca A, Urrutia A, Cancino M, Merino A, Arregui R, Rahal M. Factores de riesgo de fístula faringocutánea en laringectomía total: Experiencia del Hospital Barros Luco Trudeau 2008 a 2019. CEPE 2019, www.sochiorl.cl.

15. Öztürk K, Turhal G, Öztürk A, Kaya I, Akyıldız S, Uluöz U. The Comparative Analysis of Suture versus Linear Stapler Pharyngeal Closure in Total Laryngectomy: A Prospective Randomized Study. Turk Arch Otorhinolaryngol. 2019; 57(4): 166-70. 